\title{
Essai sur le terrain d'un vaccin atténué contre la cowdriose
}

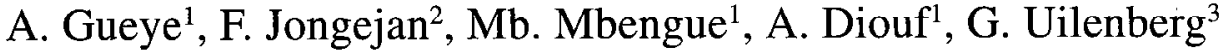

GUEYE (A.), JONGEJAN (F.), MBENGUE (Mb.), DIOUF (A.), UILENBERG (G.). Essai sur le terrain d'un vaccin atténué contre la cowdriose. Revue Élev. Méd. vét. Pays trop., 1994, 47 (4) : 401-404

\begin{abstract}
Les auteurs rapportent les résultats d'un essai sur le terrain d'une souche sénégalaise de Cowdria ruminantium atténuée par passage sur culture cellulaire. Trente moutons vaccinés et 30 moutons témoins sont introduits dans la région des Niayes au Sénégal et font l'objet d'un suivi quotidien. Dans le lot témoin, 22 cas de mortalité dus à la cowdriose sont observés dont un seul est associé à l'anaplasmose. Dans le lot vacciné, 13 animaux sont morts et Cowdria n'a été décelée que sur deux moutons qui souffraient auparavant d'ehrlichiose ou d'anaplasmose. Trois autres cas d'ehrlichiose et deux autres cas d'anaplasmose sont également enregistrés parmi ces 13 animaux. Le problème de l'interaction pathologique semble être à l'origine de la baisse de la résistance chez les deux individus vaccinés et qui présentaient des Cowdria dans le cortex cérébral. Les autres animaux du lot n'ont pas présenté de signes d'infection à Cowdria.
\end{abstract}

Mots clés : Ovin - Cowdriose - Cowdria ruminantium - Vaccin vivant Sénégal.

\section{INTRODUCTION}

L'une des contraintes pathologiques majeures à l'introduction d'animaux à haute productivité dans certaines régions tropicales est la cowdriose. Cette maladie, causée par une rickettsie, Cowdria ruminantium (Cowdry, 1925), est jusqu'à présent contrôlée, soit par une lutte systématique contre les tiques vectrices, soit par l'immunisation, en ayant recours à la méthode de l'infection suivie du traitement. Bien qu'efficace, ce dernier procédé comporte les risques d'un traitement tardif et d'une issue fatale (3). II est par ailleurs astreignant, car nécessitant un suivi quotidien des animaux jusqu'à leur traitement par des antibiotiques appropriés, en l'occurrence les tétracyclines $(3,5)$.

On a donc tenté de mettre au point un vaccin atténué (7) utilisable sur le terrain à partir d'un isolat de Cowdria ruminantium provenant de la région des Niayes (1). Ce premier essai ouvre de nouvelles perspectives de prophylaxie de cette affection. L'efficacité du vaccin démontrée au laboratoire contre le stock sauvage homologue (8), reste cependant à confirmer in situ avant d'envisager

1. ISRA, Département de Recherches sur les Productions et la Santé animales, BP 2057, Dakar, Sénégal.

2. Department of Parasitology and Tropical Veterinary Medicine, University of Utrecht, POB 80.165, 3508TD Utrecht, Pays-Bas.

3. CIRAD-EMVT, 10 rue Pierre Curie, 94704 Maisons-Alfort Cedex, France.

Reçu le 11.3.1994, accepté le 1.2.1995. son utilisation à grande échelle. Ce contrôle fait l'objet de la présente étude. Le lieu expérimental choisi est une région où l'infection sévit sous forme hyperenzootique (1, $2,4)$ en raison de la présence importante de la tique vectrice Amblyomma variegatum (Fabricius, 1794) et d'un taux de transmission élevé de l'infection (6). L'essai est réalisé durant la saison sèche à la période d'intense activité des nymphes qui débute au mois de décembre (4).

\section{MATÉRIEL ET MÉTHODE}

Le test est mené successivement en station et sur le terrain. A la station, les moutons reçoivent une inoculation du stock vaccinal et font l'objet d'un suivi sérologique pour évaluation de la réponse immunitaire. Un prélèvement de sang est effectué sur tous les moutons avant l'inoculation, puis aux $15 \mathrm{e}$ et $21 \mathrm{e}$ jours après la vaccination, afin de contrôler leur état immunitaire par la méthode de l'immunofluorescence indirecte. Au bout d'un mois, ces animaux sont transférés dans la zone des Niayes et exposés à l'infection naturelle en compagnie d'un lot de moutons témoins. Ils vont au pâturage durant la journée et une supplémentation alimentaire est distribuée à leur retour à l'enclos. Ils sont examinés quotidiennement et la température rectale est prise le matin.

En cas d'hyperthermie, un frottis de sang est réalisé pour observation au laboratoire. Sur les individus morts, un frottis de cortex cérébral est effectué afin de rechercher Cowdria ruminantium dans l'endothélium des vaisseaux.

\section{Matériel animal}

Les moutons Waralé (Taoubire x Peul) utilisés proviennent de la zone sahélienne où $A$. variegatum est très rare et très localisée. Ces animaux, tous adultes et des deux sexes, sont en principe indemnes de cowdriose. Deux lots de 30 têtes sont constitués, l'un témoin (lot 1), l'autre vacciné (lot 2 ).

\section{Vaccin}

Il est constitué par des corps élémentaires du stock "Sénégal" atténués par passages sur culture de cellulesendothéliales bovines. Un $\mathrm{ml}$ de surnageant de culture du $21 \mathrm{e}$ passage est dilué dans $29 \mathrm{ml}$ de tampon de sucrose-phosphate glutamate à $\mathrm{pH} 7,0$. Chaque animal reçoit par voie intraveineuse $1 \mathrm{ml}$ de la suspension ainsi constituée (8). 
A. Gueye F. Jongejan Mb. Mbengue A. Diouf G. Uilenberg

\section{Production d'antigène}

Le même stock sénégalais de Cowdria ruminantium, entretenu sur cultures cellulaires, est utilisé comme antigène (9). Cet antigène est conservé sous forme de corps élémentaires et de morulae à $-20^{\circ} \mathrm{C}$ en aliquotes de $200 \mu \mathrm{l}$.

\section{Immunofluorescence indirecte}

Après décongélation, la suspension d'antigène est diluée au $1 / 100$ dans du PBS $(\mathrm{pH}=7,4)$ et déposée sur des lames à immunofluorescence à raison de $10 \mu \mathrm{l}$ par spot. Les lames sont séchées puis fixées avec du méthanol et utilisées immédiatement. Sur chaque spot, on dépose 10 $\mu l$ de sérum dilué au 1/80 et on incube en atmosphère humide à la température du laboratoire pendant $30 \mathrm{~min}$. Chacune des lames porte un sérum négatif et un sérum positif, dilués au 1/80. Les lames sont ensuite lavées une fois avec du PBS puis trempées dans cette solution durant $10 \mathrm{~min}$. Du sérum anti-lgG ovines conjuguées à la fluorescéine et dilué au 1/100 dans du PBS contenant 0,01 p. 100 de bleu Evans à 1 p. 100, est ajouté. Les lames sont remises en atmosphère humide pendant 30 min puis lavées et trempées comme précédemment dans du PBS. Elles sont ensuites montées avec du glycérol et examinées au microscope à immunofluorescence.

\section{RÉSULTATS}

\section{Vaccination en station}

L'analyse des sérums recueillis sur les moutons avant l'injection de la souche atténuée et aux 15 et 21 e jours après cette inoculation donne, par la technique de l'immunofluorescence indirecte, les résultats suivants :

- pour le prélèvement prévaccinal, les sérums des 30 animaux sont tous négatifs ;

- quant aux prélèvements des 15 et 21 jours, ils se sont révélés positifs pour l'ensemble de ces moutons.

Aucune mortalité n'est enregistrée au bout d'un mois de maintien de ces animaux en station.

\section{Exposition à l'infection naturelle}

Les tableaux I et II illustrent en détail le comportement des deux lots sur le terrain.

TABLEAU I Lot 1 : Lot témoin (introduction dans la région des Niayes, 3 décembre 1992).

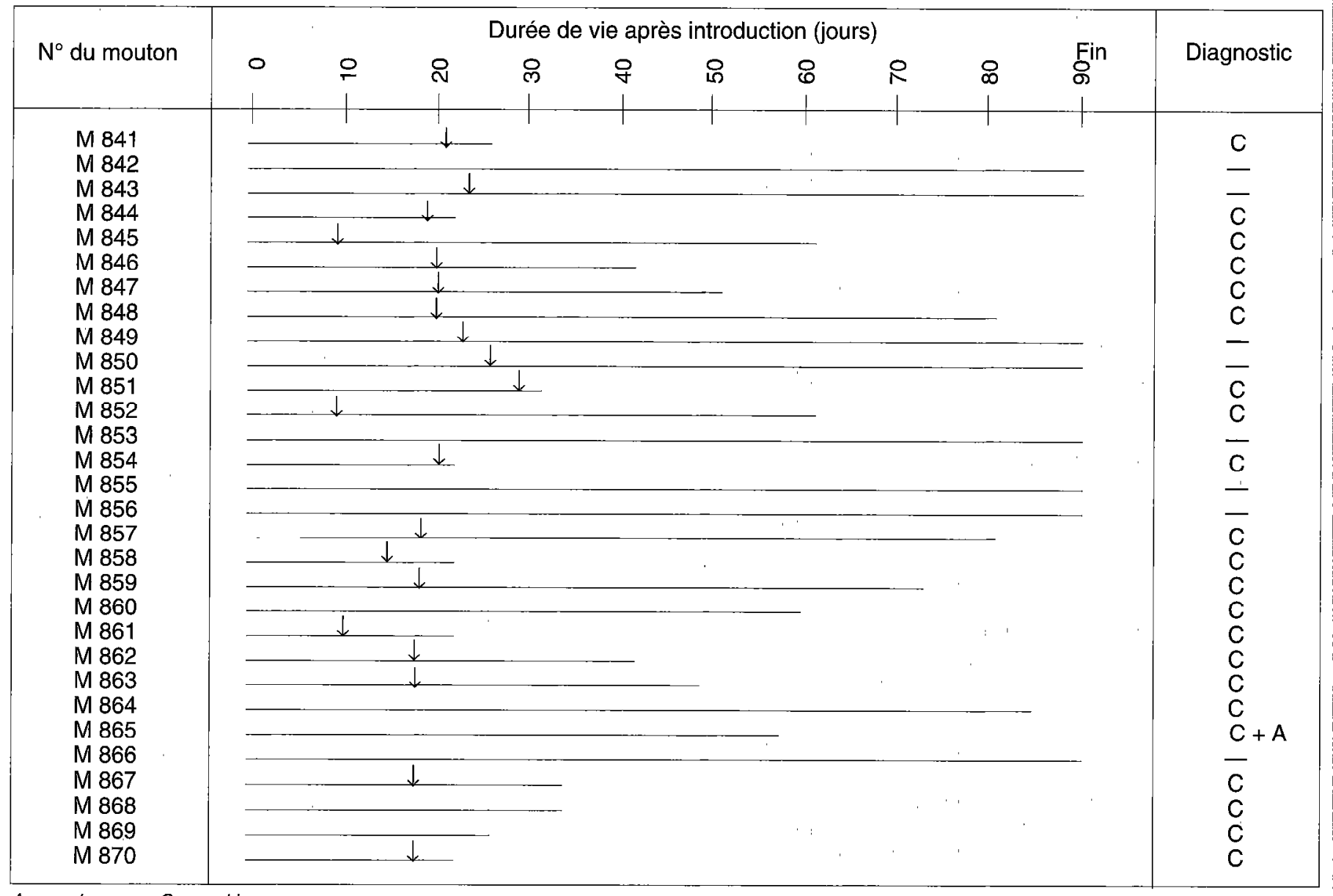


TABLEAU II Lot 2 : Moutons vaccinés (introduction dans la région des Niayes, 3 décembre 1992).

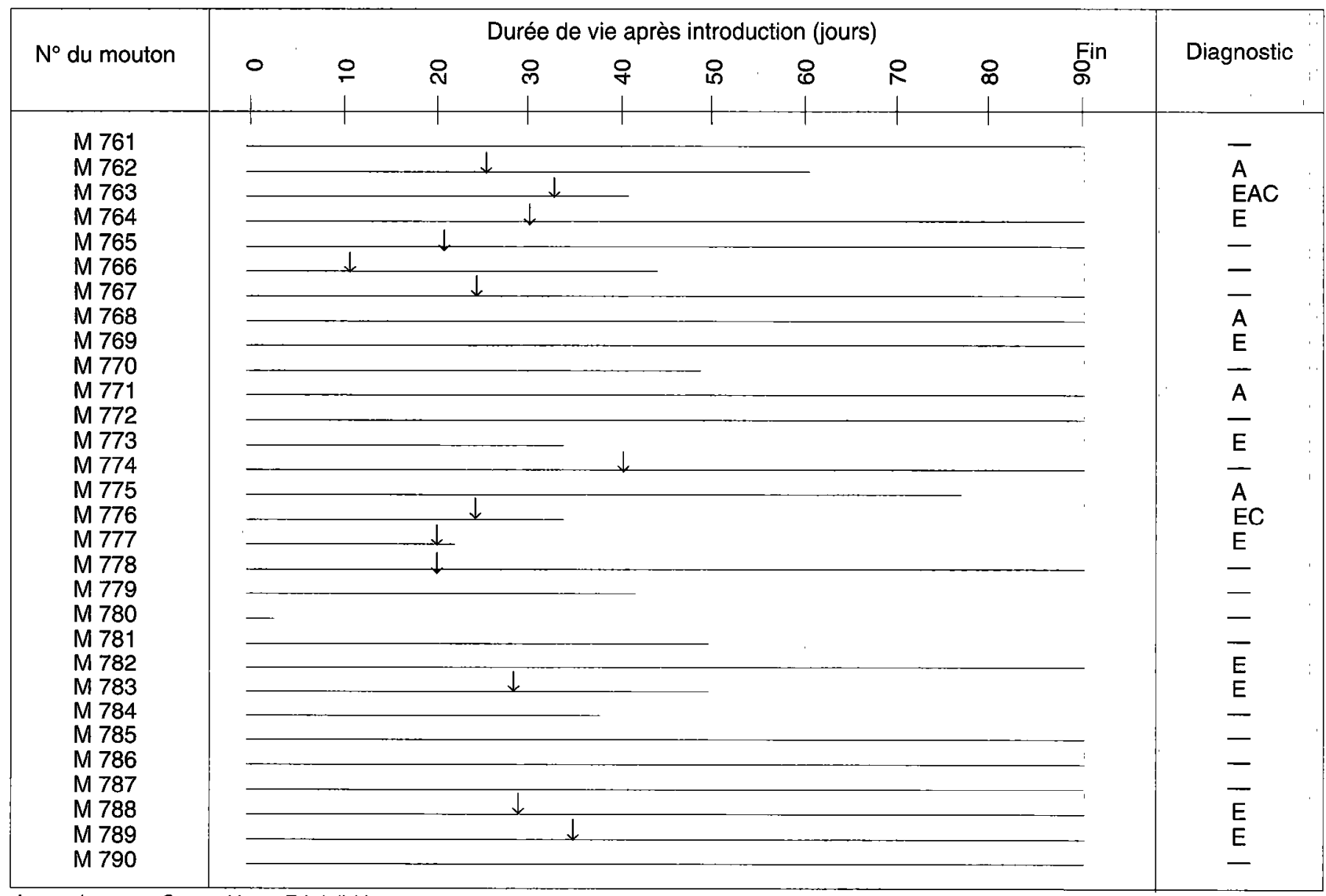

$A$ : anaplasmose; $C$ : cowdriose ; $E$ / ehrlichiose.

$\downarrow$ : début de l'hyperthermie.

\section{Lot témoin (tabl. l)}

Sur 30 moutons, seuls 8 ont survécu après leur introduction dans la zone d'enzootie. Les 22 autres individus sont morts de cowdriose.L'un d'entre eux a également montré Anaplasma ovis Di Domizio, 1919, dans son sang. Tous les cas de mortalité sont survenus dans un délai de moins de trois mois.

\section{Lot des moutons vaccinés (tabl. II)}

Sur les 30 animaux, 13 ont succombé à la suite de leur introduction dans la zone des Niayes. Au diagnostic de ces 13 moutons, il est noté : deux cas d'infection à Cowdria ruminantium associée à Ehrlichia ovina (Lestoquard et Donatien, 1936) et/ou à Anaplasma ovis (moutons $n^{\circ} 763$ et $n^{\circ} 776$ ), ainsi que trois cas d'ehrlichiose seule et deux cas d'anaplasmose seule. Pour les six autres moutons morts, aucun hémoparasite n'est décelé sur les frottis de sang, ni aucune Cowdria au niveau du cortex cérébral. Parmi les survivants, 5 ont montré $E$. ovina dans leur sang, tandis que $A$. ovis a été trouvé chez deux d'entre eux.
Le mouton $n^{\circ} 776$ ayant présenté E. ovina dans son sang pendant quatre jours, associée à une hyperthermie, est ramené à la station pour que soit isolée cette rickettsie, mais il meurt trois jours après. A l'autopsie, on note un hydrothorax important, une hydropéricardite, une légère ascite, des lésions hémorrhagiques très marquées, notamment au niveau des reins ; le frottis du cortex cérébral révèle de nombreux amas de Cowdria ruminantium. Un mouton indemne subinoculé avec le sang du $n^{\circ} 776$ présente une hyperthermie au $12 e$ jour et meurt au 15 e jour, sans que l'on puisse retrouver les Ehrtichia identifiées précédemment.

\section{DISCUSSION}

Malgré la pression pathologique due à la cowdriose dans la région des Niayes, les animaux qui ont reçu la souche atténuée supportent bien mieux l'exposition à l'infection naturelle que les individus non vaccinés. L'issue fatale des deux cas d'infection à Cowdria observés peut résulter de l'interaction avec d'autres rickettsioses, en l'occurrence l'ehrlichiose et l'anaplasmose qui ont pu réduire la capacité de résistance des moutons et favoriser la multiplica- 
tion des Cowdria. Le même phénomène a été observé sur ce site lors de travaux antérieurs relatifs à des essais d'immunisation d'un lot de moutons en pratiquant la méthode de l'infection suivie de traitement (5) ; sur 17 animaux inoculés alors avec une souche virulente puis traités, 2 individus ayant contracté l'ehrlichiose ou l'anaplasmose avaient en effet présenté à leur mort des Cowdria dans le cortex cérébral.

Les résultats de ces deux expériences d'immunisation montrent par ailleurs qu'une infection à $E$. ovina ou à $A$. ovis, bien que pathogène, n'entraîne pas forcément la prolifération de Cowdria chez un animal immunisé soumis à la transmission naturelle de la rickettsie. L'infection à Cowdria peut, en revanche, être particulièrement sévère, ce qui est bien illustré dans le cas particulier du mouton $\mathrm{n}^{\circ} 776$. Chez ce dernier animal, tout s'est passé comme si l'ehrlichiose avait préparé le lit d'une nouvelle infection à Cowdria qui s'est alors imposée comme l'affection majeure, fatale pour l'hôte.

\section{BIBLIOGRAPHIE}

1. GUEYE (A.), MBENGUE (Mb.), KEBE (B.), DIOUF (A.). Note épizootiologique sur la cowdriose bovine dans les Niayes au Sénégal. Revue Élev. Méd. vét. Pays trop., 1982, 35 (3) : 217-219.

2. GUEYE (A.), MBENGUE (Mb.), DIOUF (A.). Situation épizootiologique actuelle de la cowdriose des petits ruminants dans les Niayes du Sénégal. Revue Elev. Méd. vét. Pays trop., 1984, 37 (3) : 268-271.

3. GUEYE (A.), VASSILIADES (G.). Traitement et perspectives de chimioprophylaxie de la cowdriose ovine par une oxytétracycline à longue durée. Revue Élev. Méd. vét. Pays trop., 1985, 38 (4) : 428-432.

4. GUEYE (A.), MBENGUE (Mb.), DIOUF (A.), SEYE (M.). Tiques et hémoparasitoses du bétail au Sénégal. I. La région des Niayes. Revue Élev. Méd. vét. Pays trop., 1986, 39 (3-4) : 381-393.

5. GUEYE (A.), MBENGUE (Mb.), DIOUF (A.), VASSILIADES (G.). Prophylaxie de la cowdriose et observation sur la pathologie ovine dans la région des Niayes. Revue Élev. Méd. vét. Pays trop., 1989, 42 (4) : 497-503.

GUEYE (A.), JONGEJAN (F.), MBENGUE (Mb.), DIOUF (A.), UILENBERG (G.). Field trial of an attenuated vaccine against heartwater. Revue Élev. Méd. vét. Pays trop., 1994, 47 (4):401-404

The results are given of a field trial using a Senegalese stock of Cowdria ruminantium which had been attenuated by passage in cell culture. Thirty vaccinated and thirty control sheep were exposed in the Niayes region of Senegal and were monitored daily. In the control group, 22 animals died of heartwater, associated in one case with anaplasmosis. In the vaccinated group, 13 animals died ; Cowdria was found only in two sheep which had previously suffered from ehrlichiosis or anaplasmosis ; three other cases of ehrlichiosis and two of anaplasmosis were also observed among these 13 animals. The resistance of the two vaccinated animals which showed Cowdria in their cerebral cortex was apparently lowered by the intercurrent infections. The other animals of the vaccinated group showed no evidence of Cowdria infection.

Key words : Sheep - Heartwater - Cowdria ruminantium - Live vaccine Senegal.
On sait par ailleurs que le vaccin atténué ne résout pas les problèmes posés par la diversité antigénique de $C$. ruminantium, comme l'ont montré JONGEJAN et al. (8).

Des cas de mortalité, dont l'étiologie reste imprécise, sont en outre enregistrés lors d'introduction de moutons de la zone sahélienne dans la région des Niayes plus humide et plus froide (5).

\section{CONCLUSION}

La souche atténuée de $C$. ruminantium confère une immunité aux moutons exposés à l'infection naturelle dans la région d'origine de la souche. Cette protection est' cependant à elle seule insuffisante pour assurer la survie des animaux dans des zones écologiques comparables à celle des Niayes, où prolifèrent de nombreux vecteurs capables de transmettre diverses rickettsioses. La vaccination, combinée à une réduction stratégique des tiques, peut contribuer à consolider la stabilité enzootique vis-à-vis de la cowdriose en même temps que seront minimisées les pertes directement liées à l'infestation.

6. GUEYE (A.), MBENGUE (Mb.), DIOUF (A.). Epidémiológie de la cowdriose au Sénégal. I. Etude de la transmission et du taux d'infection d'Amblyomma variegatum (Fabricius, 1794) dans la région des Niayes. Revue Elev. Méd. vét. Pays trop., 1993, 46 (3) : 441-447.

7. JONGEJAN (F.). Protective immunity to heartwater (Cowdria ruminantium infection) is acquired after vaccination with in vitro attenuated rickettsiae. Infect. Immun., 1991, 59: 729-731.

8. JONGEJAN (F.), VOGEL (S.W.), GUEYE (A.), ULENBERG (G.). Vaccination against heartwater using in vitro attenuated Cowdria ruminantium organisms. Revue Élev. Méd. vét. Pays trop., 1993, 46 (1-2) : 223-227.

9. MARTINEZ (D.), SWINKELS (J.), CAMUS (E.), JONGEJAN (F.) Comparaison de trois antigènes pour le sérodiagnostic de la cowdriose par immunofluorescence indirecte. Revue Élev. Méd. vét. Pays trop., 1990, 43 (2) : 159-166.

GUEYE (A.), JONGEJAN (F), MBENGUE (Mb.), DIOUF (A.), UILENBERG (G.). Prueba de campo de una vacuna atenuada contra la cowdriosis. Revue Élev. Méd. vét. Pays trop., 1994, 47 (4) : 401-404

Los autores reportan los resultados de un experimento de campo con una cepa senegalesa de Cowdria ruminantium atenuada por pasages sobre cultivo celular. Treinta corderos vacunados y treinta testigos fueron introducidos en la región de Niayes, Senegal, donde fueron seguidos en forma cotidiana. En el grupo testigo, se observaron 22 casos de mortalidad a causa de la cowdriosis con un solo caso asociado a la anaplasmosis. En el grupo vacunado, 13 animales murieron, pudiendose demostrar la presencia de Cowdria unicamente en dos corderos, los cuales sufrían anteriormente de erliquiosis o de anaplasmosis. Otros tres casos de erliquiosis y dos de anaplasmosis fueron igualmente registrados entre estos 13 animales. El problema de la interacción patológica parece ser la causa de la disminución de la resistencia en los dos individuos vacunados que presentaron Cowdria en la corteza cerebral. Los otros animales del grupo no presentaron síntomass de infección por Cowdria.

Palabras clave : Ovino - Cowdriosis - Cowdria ruminantium - Vacuna viva Senegal. 